J Neurol Neurosurg Psychiatry 2004;75(Suppl IV):iv2-iv1 1. doi: 10.1136/jnnp.2004.055293

r he detail to which a neuro-ophthalmological examination can be undertaken clearly depends on the facilities available to the examiner. Examination in a fully furnished ophthalmology suite is entirely different to bedside examination with nothing but a Snellen chart, a red pin, and an ophthalmoscope. In this article we will try to provide a balance between the "ideal" and what is practically possible to the "jobbing neurologist". There are certain techniques and tools which are particularly related to the assessment of children (for example, in relation to amblyopia or squint). These will not be dealt with here as the focus of this article is the assessment of the adult. The interested reader is referred elsewhere. ${ }^{1}$

\title{
THE NEURO-OPHTHALMOLOGICAL EXAMINATION
}

\section{General examination}

The neuro-ophthalmological examination should not be seen as an independent process, but complementary to both full general examination and full neurological examination. For example, it may be that general problems such as symptoms and signs of acromegaly prompt a neuroophthalmological assessment. Alternatively, it may be the other way round as in the case of a patient presenting with amaurosis fugax who will need a full neurological examination as well as a cardiovascular assessment.

\section{Examination of head and neck}

Examination of the neck is important with respect to mass lesions and more commonly extracranial vascular occlusive disease. Leaving this aside, of particular importance is the examination of the cranial nerves which may help to localise the neurological lesion. For example, a sixth nerve palsy which is accompanied by ipsilateral numbness of the face, facial weakness, and hearing loss is likely to be caused by a lesion in the cerebello-pontine angle. Other physical signs in the head and neck are occasionally useful, and a few specific examples are listed in box 1.

\section{Examination of orbits, eyelids, and pupils}

The orbits should be inspected and auscultated. Proptosis can be assessed clinically by estimating the amount of sclera visible above and below the limbus (corneal edge). Lid retraction is assessed by the amount visible above it. More accurately, proptosis is assessed using an exophthalmometer which measures the distance between the front of the cornea and the orbital rim for each eye (fig 1). The normal range for this is less than 21-24 $\mathrm{mm}$ (depending on race and sex), but the important thing is that the measurements for the two eyes should be within $2 \mathrm{~mm}$ of each other.

The range of eyelid movement should be assessed by measuring the palpebral aperture with the eyes looking straight ahead, looking up, and looking down. In true ptosis caused by weakness of the levator palpebrae, elevation of the eyes is unlikely to increase the aperture very much, whereas the upper lid will still elevate, at least partially, if the problem is simply "senile ptosis" caused by dehiscence of the tarsal plate.

Pupillary assessment involves eliciting direct and consensual responses to light on both sides, checking for an afferent pupillary defect, and examining the response to convergence/ accommodation. The best way to do this is to have the patient fixate on an object at least $3 \mathrm{~m}$ away, and then shine a light at one eye, approaching it from slightly below. This prevents the

See end of article for authors' affiliations

Correspondence to: Associate Professor Christian Lueck, Department of Neurology, The Canberra Hospital, PO Box 11, Woden, ACT, 2606, Australia; christian.lueck@act.gov.au patient looking at the light source which could generate accommodation and thus unwanted pupillary constriction. By shining the light twice in the right eye, and twice in the left, both direct and consensual responses of each eye can be determined. The response to accommodation is easily assessed by getting the patient to look back and forth between a target held $30 \mathrm{~cm}$ in front of the face and the target $3 \mathrm{~m}$ away. Features of various efferent pupillary abnormalities are given in table 1 .

The "swinging torch test" to detect an afferent pupillary defect is, in our experience, often performed incorrectly. The test can be considered as being designed to compare the "amount of light getting into the brain" through each eye/optic nerve by detecting a change in the size of the 


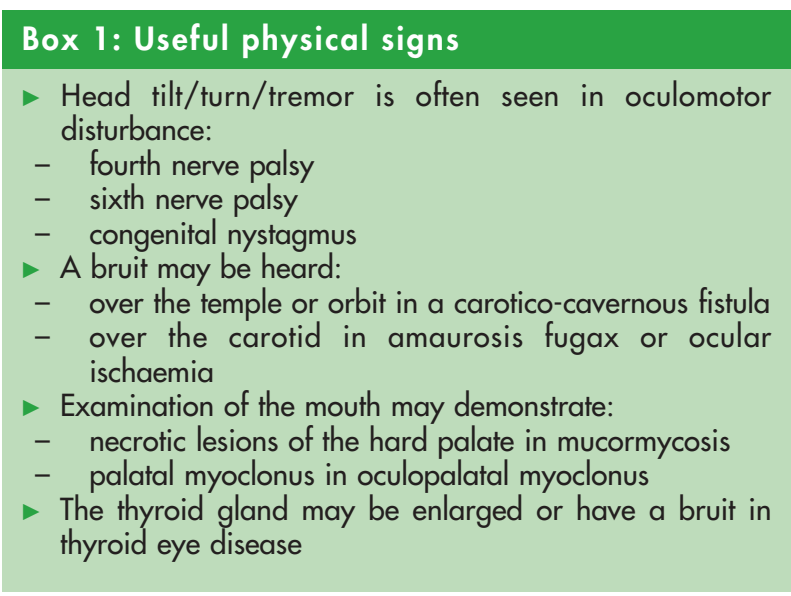

pupils as the light source is moved back and forth between the two eyes. It is not uncommon to see examiners shine the light in one eye, then swing it down and around, underneath the nose, and up to the other eye, then back down again, and so on. This is not correct. The nose/teeth are not photosensitive, and there is therefore likely to be a massive dilatation of both pupils as light is withdrawn from both eyes. This massive dilatation prevents the direct comparison of subtle differences in pupillary size which might result when the light is shone in the right as opposed to the left eye. The correct way to perform the "swinging torch test" is to shine the light in one eye, then quickly "flick" it across to the other eye, wait a second or two, then "flick" it back, and so on.

While discussing the pupils, it is worth mentioning the photostress test which is occasionally useful. If an extremely bright light is shone into an eye for 60 seconds, this will be followed by a period of reduced vision as the eye readapts to the normal ambient illumination. This can be assessed by measuring the time taken for the patient to return to their baseline Snellen acuity ( see below). This is normally achieved within 60 seconds. Prolongation occurs in global ocular ischaemia caused by severe carotid stenosis, for example, but more commonly occurs as a result of retinal pigmentary malfunction in retinal oedema, central serous maculopathy, or other macular lesions.

\section{Examination of vision}

The measurement of visual acuity and visual fields is fundamental to the assessment of visual function. Because

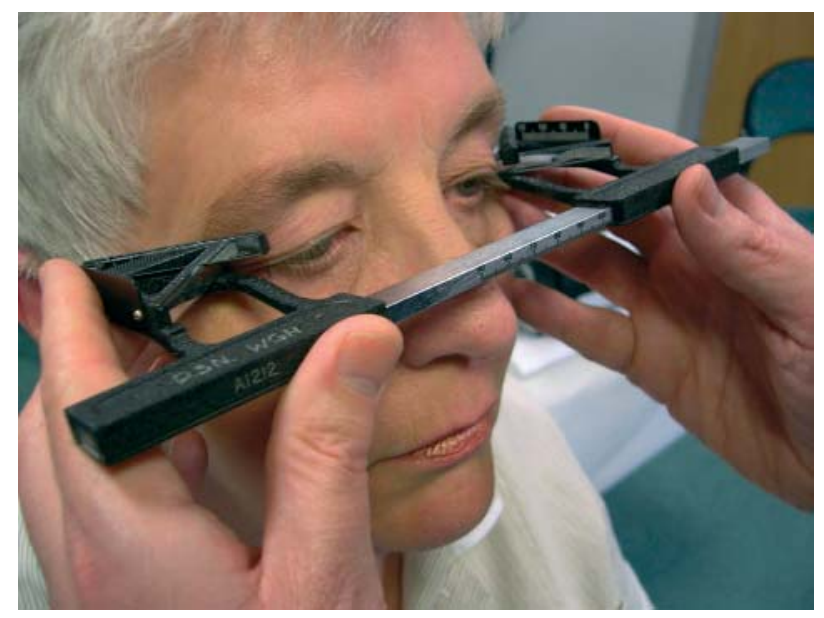

Figure 1 Hertel exophthalmometer in use. Reproduced with the permission of the patient.

vision is such a complex process, it is impossible to reduce it to a simple numerical scale without losing an enormous amount of information. However, too much information becomes unmanageable in day-to-day practice. Hence, a balance must be achieved between detecting significant departures from "normal", and not getting carried away by too much functionally irrelevant detail. Accordingly, many different methods have been proposed to measure these parameters. We will deal with the most commonly used tools in detail, and merely refer the interested reader on to obtain information on the alternatives.

\begin{abstract}
Visual acuity
It is important to assess both distance vision and near vision, as these can be affected independently by various processes, and clearly have different implications for visual disability and therefore for appropriate treatment. By far the most widely used tool for measuring distance visual acuity is the Snellen chart which effectively compares what a patient can see to what a normal person could see at $6 \mathrm{~m} \mathrm{(20} \mathrm{feet} \mathrm{in} \mathrm{the}$ USA). A normal foveal acuity should allow resolution of one minute of arc, and the letters on the 6/6 line are designed to test this. In fact, most normal people below the age of 40 years can see better than this, and will be able to read the $6 / 5$ or 6/4 lines without difficulty. In older people, an acuity of 6/9 or better is generally accepted as within normal limits, but anything worse than this requires explanation.
\end{abstract}

Table 1 Typical efferent pupillary abnormalities

\begin{tabular}{|c|c|c|c|}
\hline Abnormality & Pupillary abnormality & Associated clinical features & Pharmacological tests \\
\hline Third nerve palsy & $\begin{array}{l}\text { Pupil dilated, no response to } \\
\text { light or to accommodation }\end{array}$ & $\begin{array}{l}\text { Weakness of ipsilateral MR, IO, IR, and } \\
\text { SR. Ptosis (may be partial or complete) }\end{array}$ & - \\
\hline Horner's syndrome & $\begin{array}{l}\text { Constricted pupil, limited or no } \\
\text { response to light or to accommodation }\end{array}$ & $\begin{array}{l}\text { Ipsilateral partial ptosis. Ipsilateral } \\
\text { anhidrosis }\end{array}$ & $\begin{array}{l}10 \% \text { cocaine dilates normal pupil, not } \\
\text { sympathetically denervated one. } 1 \% \\
\text { hydroxy-amphetamine dilates pupil in } \\
\text { first or second order neurone damage }\end{array}$ \\
\hline Tonic pupil & $\begin{array}{l}\text { Dilated pupil slowly constricts to } \\
\text { accommodation, not light. Vermiform } \\
\text { movements visible on slit lamp }\end{array}$ & $\begin{array}{l}\text { Generalised areflexia in Holmes-Adie } \\
\text { syndrome }\end{array}$ & $\begin{array}{l}\text { Dilute pilocarpine }(0.125 \%) \text { constricts } \\
\text { affected pupil }\end{array}$ \\
\hline $\begin{array}{l}\text { Argyll-Robertson } \\
\text { pupil }\end{array}$ & $\begin{array}{l}\text { Small, horizontally elongated pupil. } \\
\text { No response to light, but may show } \\
\text { response to accommodation }\end{array}$ & $\begin{array}{l}\text { May be other systemic features of } \\
\text { diabetes or syphilis }\end{array}$ & - \\
\hline
\end{tabular}

$1 O$, inferior oblique; IR, inferior rectus; $M R$, medial rectus; $S R$, superior rectus. 

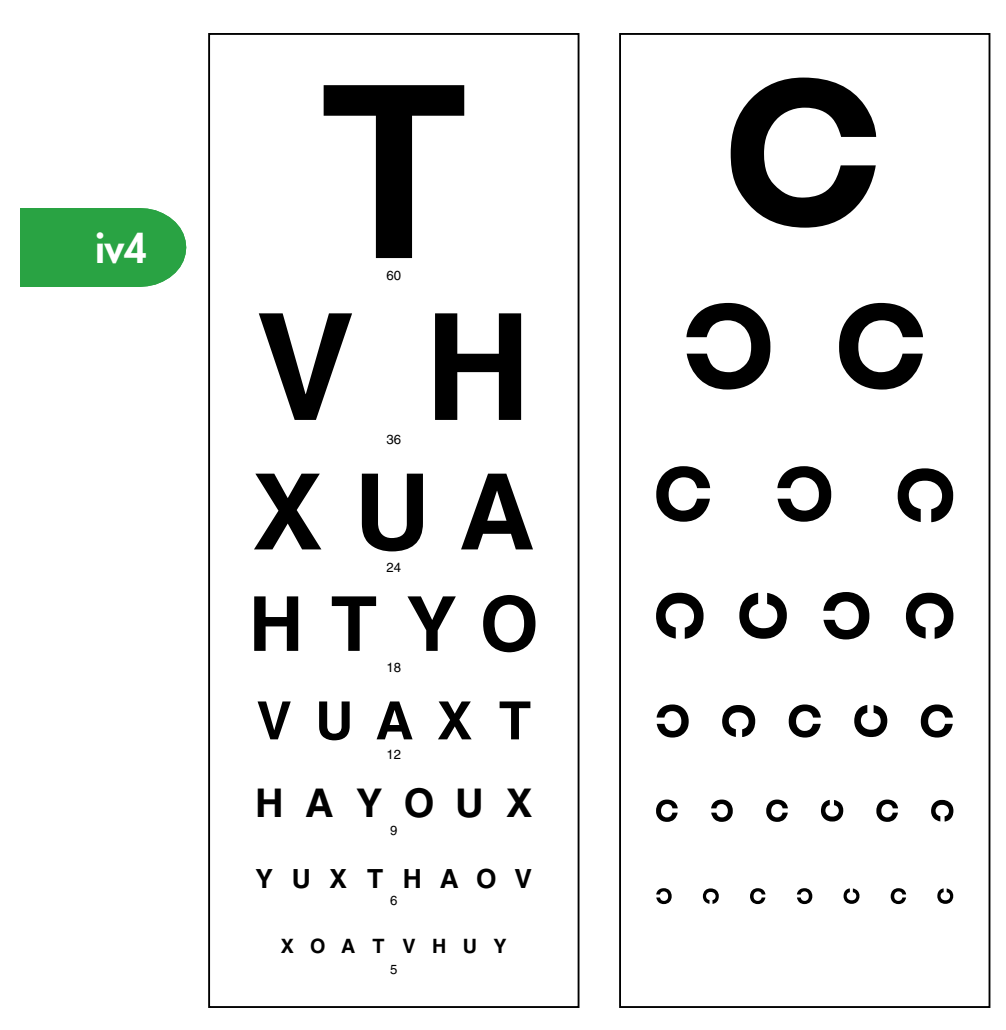

Standard Snellen acuity is assessed in relation to a $6 \mathrm{~m}$ viewing distance (for example, $6 / 18$ ), or to a viewing distance of 20 feet (for example, 20/20). The worst numerical value on the chart is $6 / 60$ (or 20/200). If the vision is worse than this, a measurable acuity can occasionally be obtained if the chart is brought nearer. For example, if the patient is able to see the top letter (officially $6 / 60$ ) at $3 \mathrm{~m}$ rather than $6 \mathrm{~m}$, this is referred to as $3 / 60$. If the vision is still worse, it is generally classified as "counting fingers" (CF), "hand movements" (HM), "perception of light" (PL), or "no perception of light" (NPL). Snellen acuities are sometimes represented on a decimal scale (for example, $6 / 6$ is $1.0,6 / 24$ is 0.25 , etc).

Not all patients can read, and so a number of variations on the Snellen chart have been designed over the years, including "Landolt C" and "Illiterate E" charts (fig 2). These require the patient to determine the orientation of the relevant letter. Another variation used for children involves matching pictures which are presented in varying sizes.

If a patient is unable to achieve an uncorrected (without help from spectacles) distance acuity of $6 / 6$ or better, the first question which needs to be answered is whether or not this relates to a refractive error in the eye-that is, can the acuity be improved by an appropriate refractive correction? In order to assess this formally, it is necessary to perform a full refraction on the patient. This resource may or may not be available. If not, use of a pin hole should be considered. The depth of focus of the eye is increased by reducing the pupillary aperture to the size of a pin hole, but this is achieved at the cost of reduction in the total amount of light entering the eye. In practical terms, a pin hole can be expected to improve the acuity by about four lines of the Snellen chart. It is important to remember that even if the patient brings their own spectacles, these may not provide the best possible refractive correction for a large number of possible reasons.

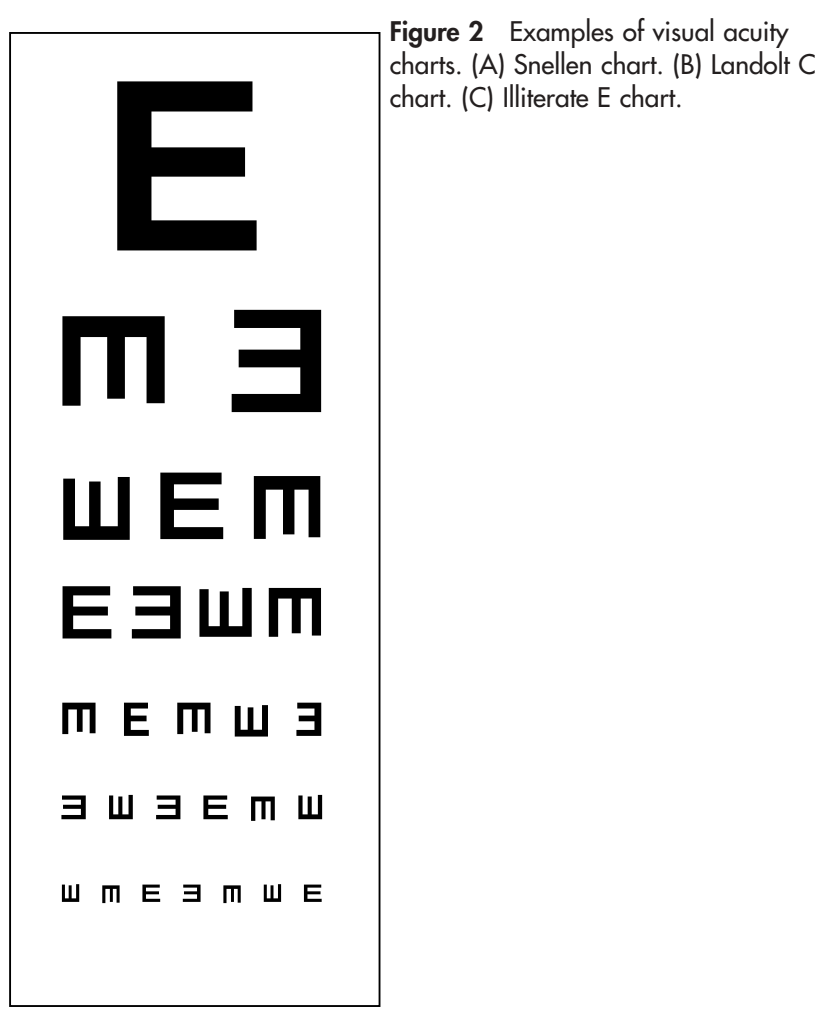

Near visual acuity is assessed using pre-printed text sizesfor example, the Jaeger near vision reading charts. The text is held approximately $30 \mathrm{~cm}$ from the eye and the patient is asked to read the smallest text possible. Simple presbyopia will obviously impair near acuity while distance acuity is preserved, but a similar effect may be observed with, for example, cataract-pupillary constriction associated with accommodation reduces overall light entry and increases the "obscuring" effect of the cataract. Obviously, both eyes must be assessed independently for both distance and near vision, as well as for colour vision.

\section{Colour vision}

In day-to-day practice, colour vision is usually assessed using Ishihara pseudo-isochromatic test plates. These were designed to detect inherited colour blindness which results in the patient seeing a different number from that seen by a person with normal colour vision. In neuro-ophthalmological practice, lack of colour vision is generally determined by the inability to see a number at all. This is obviously relatively crude. More accurate assessment of colour vision can be obtained with the Hardy-Rand-Ritter (HRR) charts, or with the Farnsworth-Munsell 100 hue test (fig 3). Both of these involve getting the patient to compare colours which are subtly different to obtain the "nearest match". The latter in particular takes a very long time to perform and is therefore not suited to routine use in most busy clinics.

\section{Depth perception}

Binocular depth perception (stereopsis) can be assessed by the use of a number of variations on the theme of random dot stereograms or, even more formally, by the use of a device called the synoptophore. In general, however, this is used for assessing children with amblyopia or strabismus, and does not form part of the routine assessment of adult patients. 

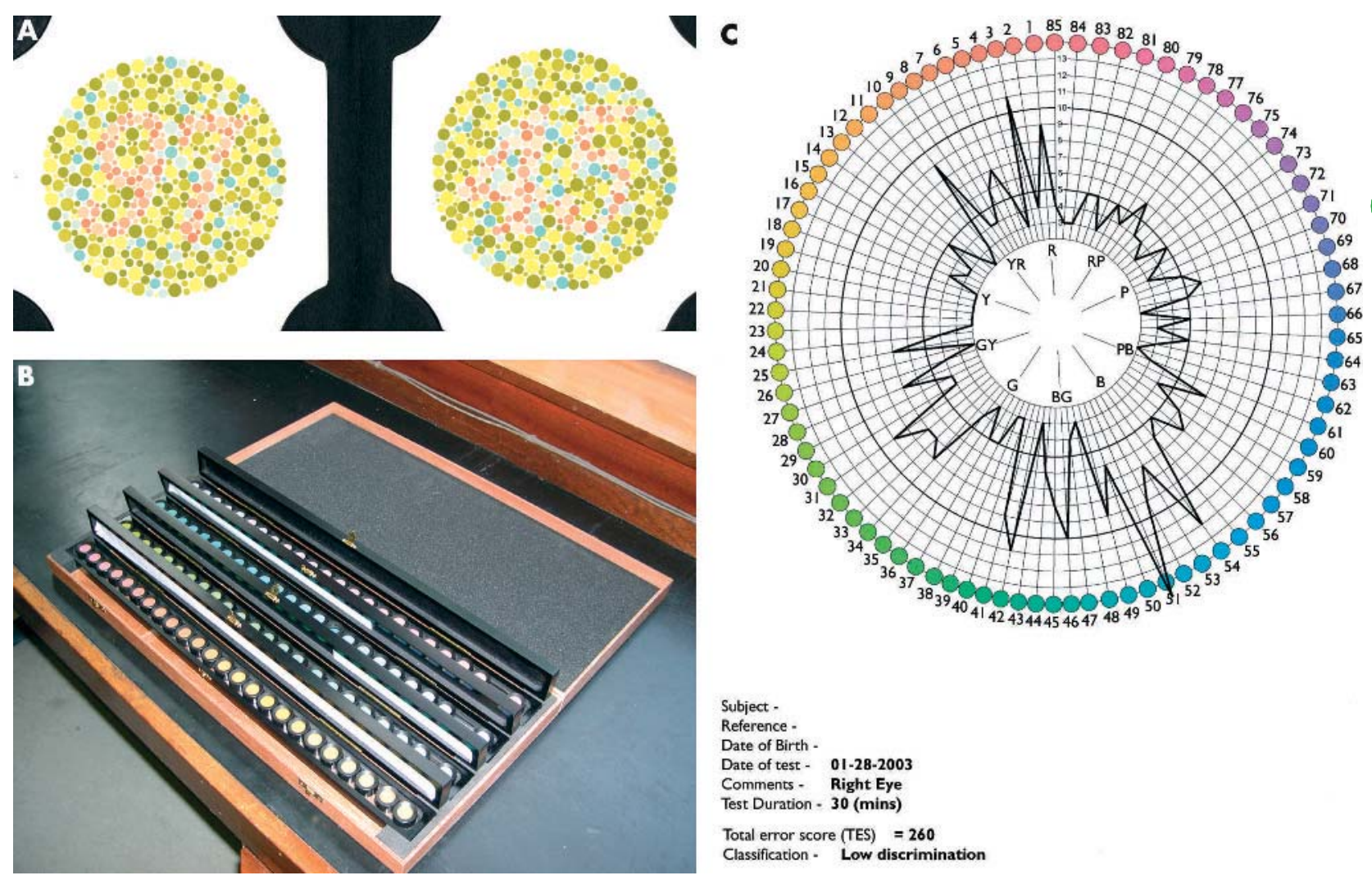

Subject -

Reference -

Date of Birth .

Date of test - 01-28-2003

Comments - Right Eye

Test Duration - $\mathbf{3 0}$ (mins)

Total error score (TES) $\mathbf{2} \mathbf{2 6 0}$

Classification - Low discrimination

Figure 3 Techniques for assessing colour vision. (A) Ishihara test plate. (B) Testing counters for Farnsworth-Munsell 100 Hue test. (C) Result of 100 Hue test, showing tritan (blue-yellow) defect.

\section{Visual fields}

These can be assessed in a number of different ways, ranging from bedside confrontation to formal automated perimetry. At bedside level, several different methods of confrontation exist, ranging from waggling fingers in the periphery to using a small red pin. Most neurologists and neuro-ophthalmologists have their own "pet" way of doing things which was fair enough in the absence of evidence suggesting that any one way was better than another. Recently, however, Pandit et al ${ }^{2}$ have compared the various techniques in a prospective manner. They found that by far the most sensitive technique was the use of a small $(5 \mathrm{~mm})$ red target, specifically asking the patient when the colour became detectable, not just the pin itself. This generates a visual field of about $\pm 20^{\circ}$, which is a manageable size and allows the examiner to compare a patient's visual field directly with his or her own. Detection of scotomata (including measuring the size of the blind spot) is relatively straightforward using this technique. We strongly suggest that all practitioners adopt this method and dispense with "waggling fingers".

If available, the Bjerrum screen provides a useful method of assessing visual fields. The subject is seated at an appropriate distance in front of an appropriate sized grid whose circular markings signify the $10^{\circ}$ steps away from fixation. A number of radial axes indicate the horizontal, the vertical, and a number of intervening steps. Targets of different sizes and colours can be brought in from the periphery (either physically or by projection with a hand held device) while the subject fixates a central point. The threshold for detection is determined for each radius, thereby mapping out an isopter (a line joining points of equal sensitivity in the visual field-rather like a contour on a map). By flashing a projected target at different points in the visual field, it is possible to detect and map out small scotomas.

When mapping out scotomas, either by confrontation or on the Bjerrum screen, it is generally easier to move the target from blind field into seeing field rather than the other way round.

Many different methods have been developed for more formal measurement and documentation of visual fields. These can broadly be divided into devices which use kinetic or static approaches. The kinetic approach involves determining the detection threshold when a target is brought in from the periphery until it can just be seen. The target is generally moved around the visual field, and the successive detection points are eventually joined together to generate an isopter as above. By varying the size and brightness of the target, several different isopters can be obtained, generating what is effectively a contour map of vision (fig 4). The most widely used perimeter of this sort is the Goldmann perimeter.

Kinetic perimetry is not as sensitive as static perimetry at detecting small areas of visual field loss of the sort which happen in glaucoma, for example. In static perimetry, a target at a given position in the visual field is gradually increased in intensity until it can just be detected. By looking at a large number of points in the visual field, it is again possible to provide a description of what the patient can or cannot see. Because this technique generates numbers, it is possible to perform mathematical manipulation, and so generate statistical comparisons with predicted normal vision. This process is automated, and the most widely used devices of this sort 


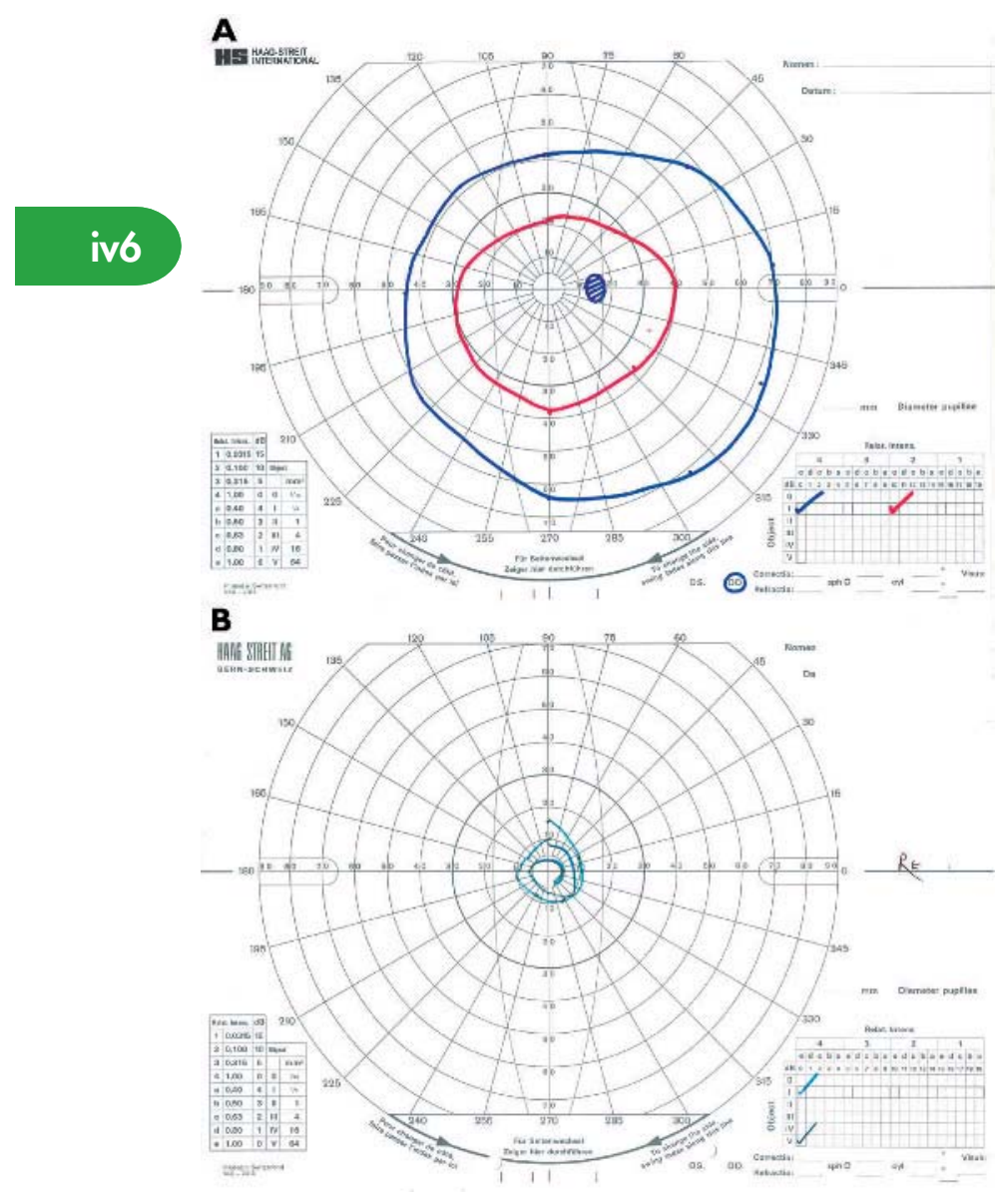

Figure 4 Plots of visual fields derived from Goldmann perimeter. (A) Normal visual field demonstrating normal isopters. (B) "Spiral visual field" and crossing isopters seen in functional elaboration.

are the Humphrey, the Octopus, and the Medmont perimeters. An example of a Humphrey printout is shown in fig 5 .

Interpreting the output of automated perimeters is potentially very daunting as the page is covered with small numbers and little grey boxes. A few pointers to help in the interpretation are given in box 2 .

There are advantages and disadvantages of dynamic and static methods for assessing visual fields. An overview is provided in table 2 .

Several alternative techniques for estimating visual fields have recently been developed based on different physiological properties of the visual pathways. These include short wavelength automated perimetry, high pass resolution perimetry, flicker perimetry, motion and displacement perimetry, and frequency doubling perimetry. Unfortunately, space does not permit detailed discussion here. The interested reader is referred to the chapter in Walsh and Hoyt ${ }^{3}$ in the first instance.

\section{Examination of the eye}

On the ward, or in a standard neurological outpatient clinic, the only available tool will be the direct ophthalmoscope. In a neuro-ophthalmological clinic, a slit lamp (fig 6) will usually be available, allowing examination of the eye in much greater detail. With just an ophthalmoscope, examination of each eye should consist initially of confirming that there is a red reflex

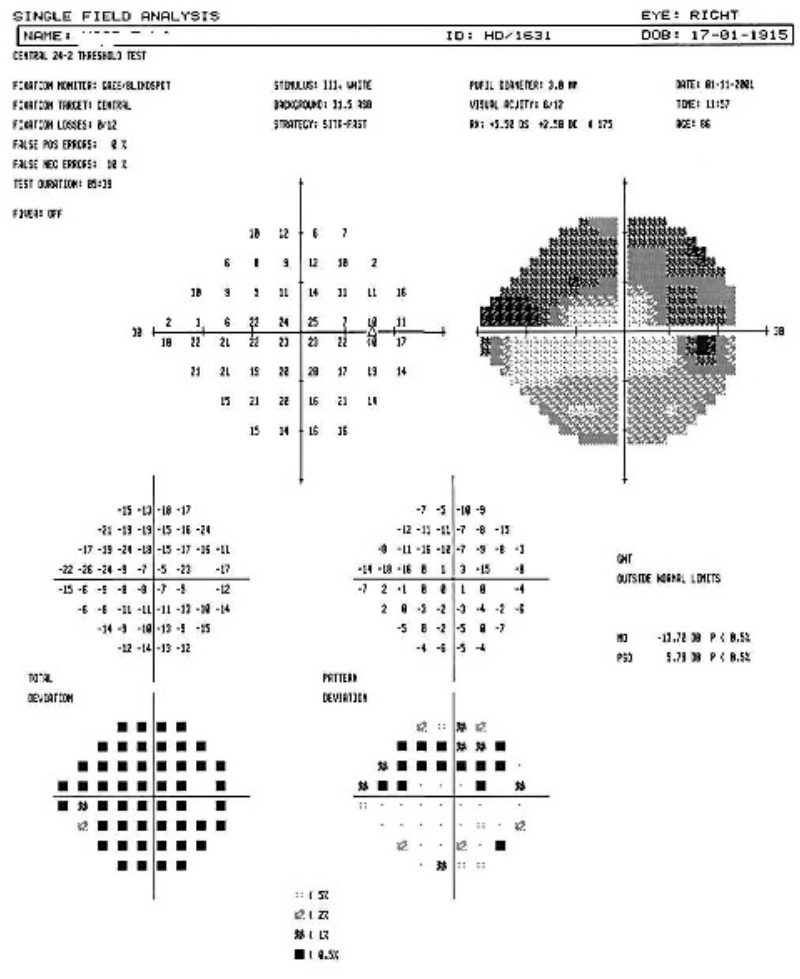

Figure 5 Plot of visual field derived from Humphrey perimeter showing large arcuate scotoma in superior visual field. Note that there is global reduction in detection threshold caused by a mild cataract. When the total deviation (bottom left) is corrected for this to generate the pattern deviation (bottom right), the true nature of the scotoma is revealed.

and gauging the quality of the refractive media by the clarity with which the retina can be imaged. The optic fundus should be found and assessed with regard to the points listed in box 3. This task will be made much easier by the use of mydriatic eye drops, but this is not always possible. By way of a practical tip, it should be noted that the lenses in a direct ophthalmoscope are simple spherical lenses. If the patient has a high degree of astigmatism, it will not be possible to focus on the fundus using just spherical lenses. In this situation, the best results are obtained by asking the patient to put their spectacles on and looking at the retina through their glasses.

If a slit lamp is available, assessment can be much more comprehensive. In this case, the eye itself can be examined, starting with the eyelids, working backwards from sclera and cornea, through the anterior chamber, lens and posterior chamber, to the fundus. Common abnormalities at each level are given in table 3, and a few examples are shown in fig 7 .

\section{Examination of eye movements}

This is dealt with in detail in the article by Danchaivijitr and Kennard. ${ }^{4}$ A brief overview of the different types of eye movement is provided in table 4 , and an overview of eye movement examination in table 5 .

\section{Assessment of higher visual function}

We will not discuss this further here; readers are referred to excellent reference sources. ${ }^{35}$ 
Box 2: A brief guide to the interpretation of automated perimetry printouts

(1) Check the patient name and which eye is being tested

(2) Check which test programme was used:

(a) Programmes are either "static" or "suprathreshold static". In the former, target lights are made brighter and brighter until the patient can just detect them. In the latter, target lights of fixed luminance are used, the luminance being one which most normal people would just be expected to detect. Suprathreshold tests are therefore much quicker and useful as a screening test, but provide much less accurate information

(b) The extent of the visual field tested is very variable. Most ophthalmologists use a programme which tests the central $24^{\circ}$ or $30^{\circ}$ of vision. These are not adequate for neurological purposes - automated tests assessing out to $60^{\circ}$ should be requested

(3) Ascertain how reliable the patient is at performing the test. Three assessments are usually provided:

(a) False positive tests: no target is provided

(b) False negative tests: a previously detected target is represented

(c) Fixation losses. The normal inability to see targets presented in the blind spot is used to check the subject's fixation - if the subject starts to see it during the course of the test, fixation must have moved. Note that if the subject has a large temporal scotoma (for example, hemianopia) which includes the blind spot, fixation can wander widely without detection by the machine

(4) If available, compare mean deviation results with pattern deviation results. Pattern deviation is derived by correcting mean deviation results for overall sensitivity loss. For example, if a patient has a cataract, their threshold for detection will be globally reduced. On the mean deviation ("raw data") printout, this may appear as an artefactual very large scotoma which is less obvious/absent on the pattern deviation printout. A true scotoma will be consistent in both mean and pattern deviation printouts

\section{Inconsistencies in functional disorders}

As with other aspects of neurology, it is worth bearing in mind that some patients with visual symptoms elaborate their symptomatology either consciously or subconsciously, and that the pupillary responses represent the only true "objective" test of visual function. Though not common, it is important to be aware of this phenomenon. There are certain useful pointers which suggest elaboration. Perhaps most

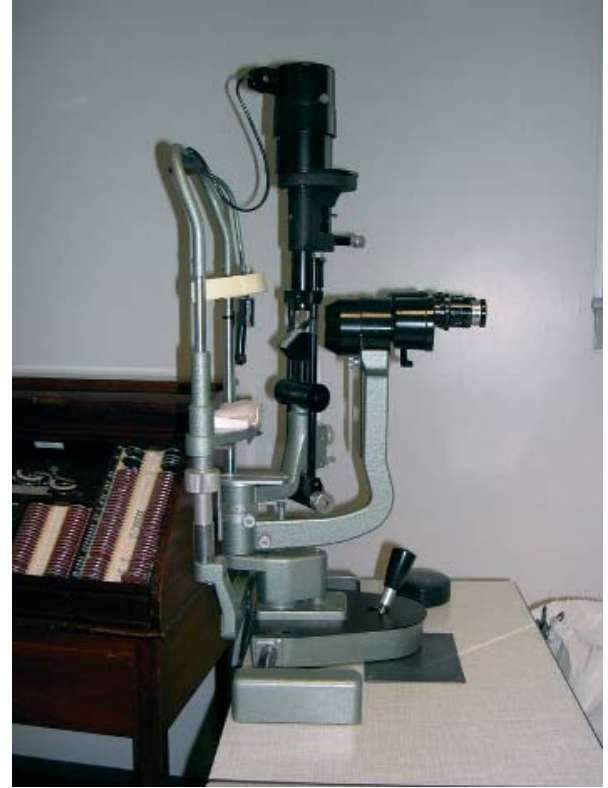

Figure 6 Haag-Streit biomicroscope (slit lamp).

important is the patient's behaviour. Is their "visuallyguided" behaviour consistent with the stated degree of visual impairment? For example, a patient who has severe tunnel vision will obviously find it difficult to walk without bumping into obstacles. An "overacting" patient may deliberately veer slightly out of their path in order to bump into something which was not actually in their way, thereby attempting to demonstrate how "blind" they are. Similarly, we have come across several patients who have "declared" visual acuities of $6 / 36$ or worse who are somehow able to look up the addresses of their local opticians in their address books when requested! Remember, however, that patients with large central scotomata (for example, in Leber's hereditary optic neuropathy) are still able to navigate well because of preserved peripheral vision in spite of having acuities of $6 / 60$ or less.

It is worth checking that the degree of optic atrophy and the pupillary responses are consistent with what appears to be going on. For example, a patient who has apparently suffered optic neuritis in the past and has been left with vision of 6/60 or worse in one eye is unlikely to have a normal (that is, not pale) optic disc in the affected eye, or not to have a demonstrable afferent pupillary defect.

Table 2 Comparison of static and kinetic perimetry

\begin{tabular}{lll}
\hline & Advantages & Disadvantages \\
\hline Kinetic perimetry (e.g. Goldmann perimeter) & Relatively simple to perform and interpret & Calibration issues \\
& Sensitive for peripheral field loss & Very user dependent and time consuming \\
& Good for detecting neurological field loss & \\
Static perimetry (e.g. Humphrey, Octopus, or & Standardised test conditions/strategies & More difficult to interpret \\
Medmont perimeters) & Numerical and statistical analysis possible & Not user independent! \\
& Less time consuming & Fixation loss in hemianopia may not be detected \\
& Automatic calibration & Not so easy to detect functional loss \\
& Very useful for ophthalmologists in & Most ophthalmologists use $24 / 2$ or $30 / 2$ programme \\
& detection of (and serial follow up of) & which may miss peripheral field loss \\
& glaucomatous visual fields & \\
\hline
\end{tabular}


Box 3 Examination of the optic fundus

- Overall shape of the disc. Is this a congenitally tilted disc?

- Overall colour of the disc. Is the disc pale? Is there pigmentary abnormality suggesting a congenital abnormality (such as a coloboma-note that the pigmented temporal crescent most often seen in myopes is normal)

- The size of the optic cup. Compared to the overall disc diameter, the cup:disc ratio is normally less than 0.7. Ratios greater than this are seen in conditions which cause nerve fibre loss - for example, glaucoma

- The vascular architecture. Do the vessels branch normally? Abnormalities such as trifurcations (as opposed to the normal bifurcations) are often associated with optic nerve head drusen.

- Evidence of disc swelling. Is there venous pulsation? Absence of pulsation is the earliest sign of papilloedema, but it is absent in $20 \%$ of normal eyes. In this case, it can often be elicited by gentle pressure on the eyeball. As the papilloedema worsens, the optic disc becomes raised, and exudates and haemorrhages may be seen

- Changes in the surrounding retina. The following should be sought within the limits of pupillary dilatation:

- hard and soft exudates

- microaneurysms and new vessel formation

- haemorrhages

- pigmentary changes (for example, "bone spicules" in retinitis pigmentosa, "salt and pepper" in mitochondrial disease)

- macular changes (for example, pigmentary changes, oedema, cherry red spot)

When examining visual fields, Goldmann perimetry may reveal spiral visual fields or crossing isopters (fig 4B). Isopters are like contours on a map-they need to join up with themselves and cannot cross. If there are inconsistencies, this indicates that the visual fields are at best uninterpretable, and at worst fabricated. Similarly, patients may occasionally attempt to "fool" automated perimeters. The reliability indices referred to in box 3 often help to detect this, but a "good" performer can outwit the machine. It is always worth being alert to this possibility.

Table 3 Examples of neuro-ophthalmologically relevant ocular abnormalities found on slit lamp examination

\begin{tabular}{|c|c|c|}
\hline Site & Abnormality & Significance \\
\hline Sclera & $\begin{array}{l}\text { Dilated blood vessels } \\
\text { ("red eye") }\end{array}$ & $\begin{array}{l}\text { Conjunctivitis, iritis, } \\
\text { carotico-cavernous fistula }\end{array}$ \\
\hline Cornea & Kayser-Fleischer rings & Wilson's disease \\
\hline $\begin{array}{l}\text { Anterior } \\
\text { chamber }\end{array}$ & Cells & Anterior uveitis \\
\hline Iris & $\begin{array}{l}\text { Synechiae } \\
\text { Lisch nodules } \\
\text { Vermiform movements }\end{array}$ & $\begin{array}{l}\text { Anterior uveitis } \\
\text { Neurofibromatosis type } 1 \\
\text { Tonic pupil }\end{array}$ \\
\hline Lens & Opacity (cataract) & $\begin{array}{l}\text { Many possible causes, } \\
\text { including diabetes and } \\
\text { myotonic dystrophy }\end{array}$ \\
\hline \multirow{3}{*}{$\begin{array}{l}\text { Posterior } \\
\text { chamber }\end{array}$} & Cells & Posterior uveitis \\
\hline & Blood & Vitreous haemorrhage \\
\hline & Tethering of vitreous & Posterior vitreous detachment \\
\hline \multirow{4}{*}{$\begin{array}{l}\text { Retina } \\
\text { (peripheral } \\
\text { retina is } \\
\text { better seen) }\end{array}$} & $\begin{array}{l}\text { See all features in } \\
\text { box } 3\end{array}$ & See all features in box 3 \\
\hline & Cholesterol embolus & Large vessel atheroma \\
\hline & Vascular sheathing & Vasculitis \\
\hline & $\begin{array}{l}\text { Peripheral pigmentary } \\
\text { changes }\end{array}$ & Mitochondrial disease \\
\hline
\end{tabular}

There are many other clues to functional elaboration of visual disability. Space does not allow a detailed discussion here. The interested reader is referred elsewhere. ${ }^{6}$ Though uncommon, correct detection of this phenomenon will prevent inappropriate investigation and treatment. We have seen an unfortunate case of a lady treated with high dose immunosuppression for a year for presumed "vasculitis": her visual loss was, in fact, entirely fabricated.

\section{NEURO-OPHTHALMOLOGICAL INVESTIGATIONS Neurological investigations}

It is assumed that the reader is familiar with the various forms of investigation common in neurological practice, specifically imaging, electrophysiology, blood tests, and lumbar puncture. Only specific points relevant to neuroophthalmology will be highlighted here.

With regard to imaging, it is now generally accepted that there is no place for a standard skull $x$ ray in neuroophthalmological investigation. ${ }^{7}$ Computed tomographic (CT) and magnetic resonance imaging (MRI) scanning are appropriate investigations to look for a structural cause such as a tumour or an aneurysm. Which type of scan is used depends on a variety of factors such as the likely pathology, scanner availability, waiting time, and cost. In certain situations, CT may still have an advantage over MRI. For example, small areas of calcification are much easier to detect by CT, and this makes it particularly useful for detecting small optic nerve sheath meningiomas and calcified optic nerve head drusen. However, for the most part, MRI imaging is preferable if it can be obtained. For example, it is not unknown for pituitary adenomas to be missed by CT but detected on MRI.

CT and MR angiography have revolutionised many aspects of neuro-ophthalmological practice by removing the need for formal intra-arterial angiography. For example, a posterior communicating artery aneurysm large enough to cause an oculomotor nerve palsy will be detected on MRI scan/MR angiography. Similarly, MR venography frequently detects cerebral sinus thrombosis non-invasively.

Visual evoked potentials are not commonly used any more. MRI is vastly superior in diagnosing optic neuritis and multiple sclerosis. However, they may be useful to demonstrate the presence of an optic nerve lesion which is clinically obvious but not associated with any abnormality on MRI.

Table 4 Types of eye movement

\begin{tabular}{ll}
\hline $\begin{array}{l}\text { Type of eye } \\
\text { movement }\end{array}$ & Brief description \\
\hline Vestibular & $\begin{array}{l}\text { Slow, conjugate, movements designed to compensate } \\
\text { for head movements, thereby keeping eyes on target }\end{array}$ \\
Optokinetic & $\begin{array}{l}\text { Slow, conjugate, movements designed to use large } \\
\text { field visual information to stabilise eyes, thereby } \\
\text { assisting vestibular system which tends to fatigue after } \\
\text { a few seconds }\end{array}$ \\
& $\begin{array}{l}\text { Fast, conjugate, movements: } \\
\text { - occur spontaneously to a variable extent }\end{array}$ \\
& - reset position of eyes in orbits during \\
& vestibular and optokinetic movements
\end{tabular}



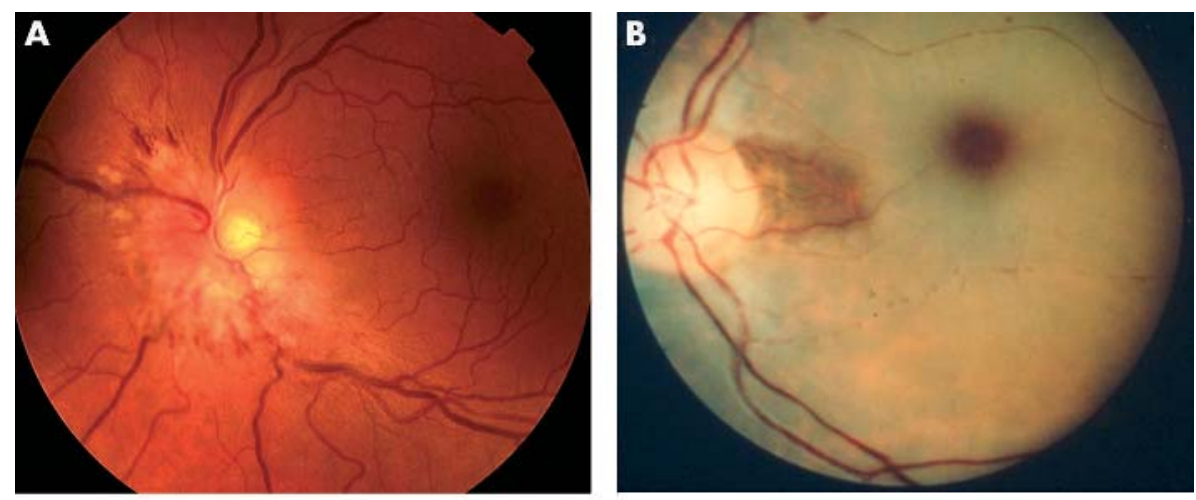

Figure 7 Ocular abnormalities.

(A) Anterior ischaemic optic

neuropathy-note swelling of the optic disc. (B) Acute central retinal artery occlusion with sparing of cilio-retinal artery-note oedematous (white) retina with cherry red spot at macula.

(C) Central retinal vein occlusion.

(D) Retinitis pigmentosa-note "bone
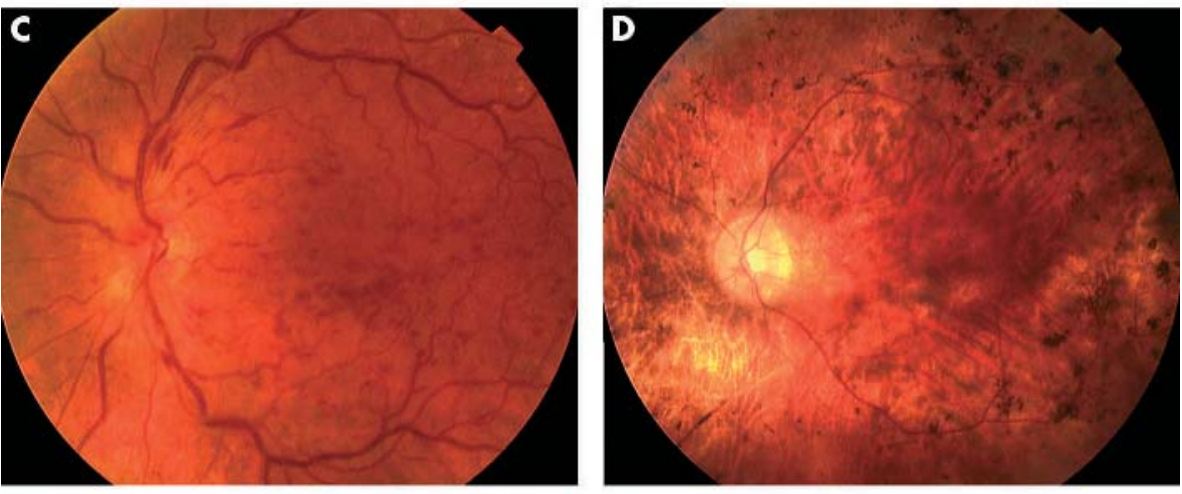

spicule" pigmentary changes. (E) Optic atrophy of temporal disc-note how pale the disc is in true optic atrophy. (F) Optic nerve head drusen-note yellow refractile bodies within discs with associated lack of optic cups.
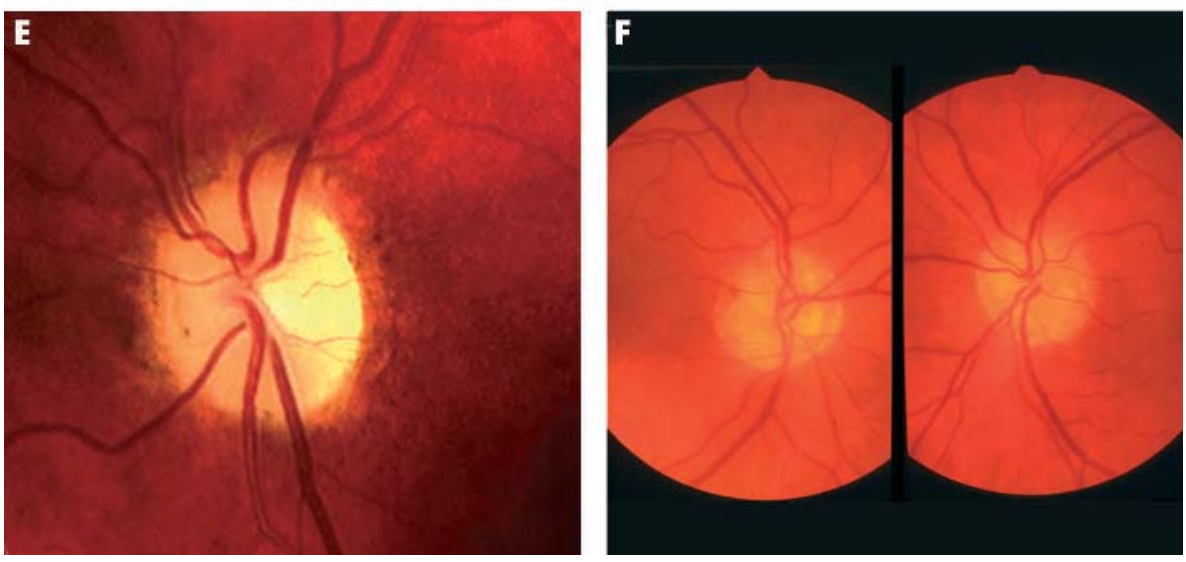

Alternatively, they may be useful to demonstrate intact visual pathways in a patient who claims to be blind but whose behaviour is not consistent with this-that is, they can be used to support the diagnosis of functional visual disturbance.

Discussing different blood tests in detail is beyond the scope of this article. Lumbar puncture, however, is worth a specific comment. This procedure is clearly useful in the diagnosis of subarachnoid haemorrhage, infections, inflammatory disease, and malignancy. It is also essential in the diagnosis of idiopathic intracranial hypertension (IIH). Many practitioners seem to feel that IIH can be diagnosed by the combination of headache, swollen discs, a normal CT scan, and normal MR venography. This is not correct. It is not unknown for overweight patients with migraine to have "pseudo-papilloedema" (for example, optic nerve head drusen) and to be misdiagnosed as having IIH, thereby resulting in inappropriate treatment. Lumbar puncture to demonstrate raised intracranial pressure is essential to prevent this. In this regard, it should also be borne in mind that the normal range of intracerebral pressure extends as high as $25 \mathrm{~cm} \mathrm{H}_{2} \mathrm{O}$ in obese subjects. ${ }^{8}$

\section{Ophthalmological investigations}

There are a few investigations which, though commonly used by ophthalmologists, are unfamiliar to most neurologists and yet potentially useful in neuro-ophthalmological practice. These include fluorescein angiography, electroretinography, and ocular ultrasound (fig 8).

Fluorescein angiography allows detailed assessment of retinal vasculature. It is capable of demonstrating areas of abnormal retinal perfusion, thereby assisting in the diagnosis of such conditions as diabetic retinopathy, vasculitis, and arteriovenous malformations (von Hippel-Lindau syndrome) as well as infective and neoplastic diseases of the retina and underlying choroid. Of particular use to the neuro-ophthalmologist is the ability to detect low grade leakage at the optic disc, thereby confirming a diagnosis of papilloedema. The process of performing fluorescein angiography requires the use of ultra-violet illumination which is what causes the dye 
Table 5 Clinical examination of eye movements (see also Danchaivijitr and Kennard ${ }^{4}$ )

\begin{tabular}{ll}
\hline Step & Comments \\
\hline $\begin{array}{l}\text { General examination } \\
\text { Orbital examination }\end{array}$ & Head postures/shaking \\
& Proptosis/enophthalmos \\
& Eyelid abnormalities \\
Pupillary abnormalities & Malalignment (cover tests) \\
Involuntary movements: & Saccadic intrusions (SWJs, MSWJs, flutter, opsoclonus) \\
& Other (e.g. myokymia, bobbing, etc) \\
& Disorders of muscle, nerve, NMJ, or central disorder \\
Versions/ductions ("'H" pattern) & Antisaccades \\
Saccades (horizontal and vertical) & Various types: \\
Nystagmus (9 cardinal positions) & Common: vestibular, cerebellar/brainstem, congenital \\
& Unusual but important: see-saw, PAN, convergence-retraction, \\
& pendular \\
& Rare: eyelid, epileptic, pursuit \\
& alternate sursumduction)
\end{tabular}

MSWJ, macro square wave jerks; NMJ, neuromuscular junction; OKN, optokinetic nystagmus; PAN, periodic alternating nystagmus; SWJ, square wave jerks.

to fluoresce. Optic nerve head drusen near the surface of the disc often exhibits the property of autofluorescence-if this is specifically looked for before the injection of the fluorescein it can be enormously helpful in distinguishing such drusen from a pathologically swollen disc.
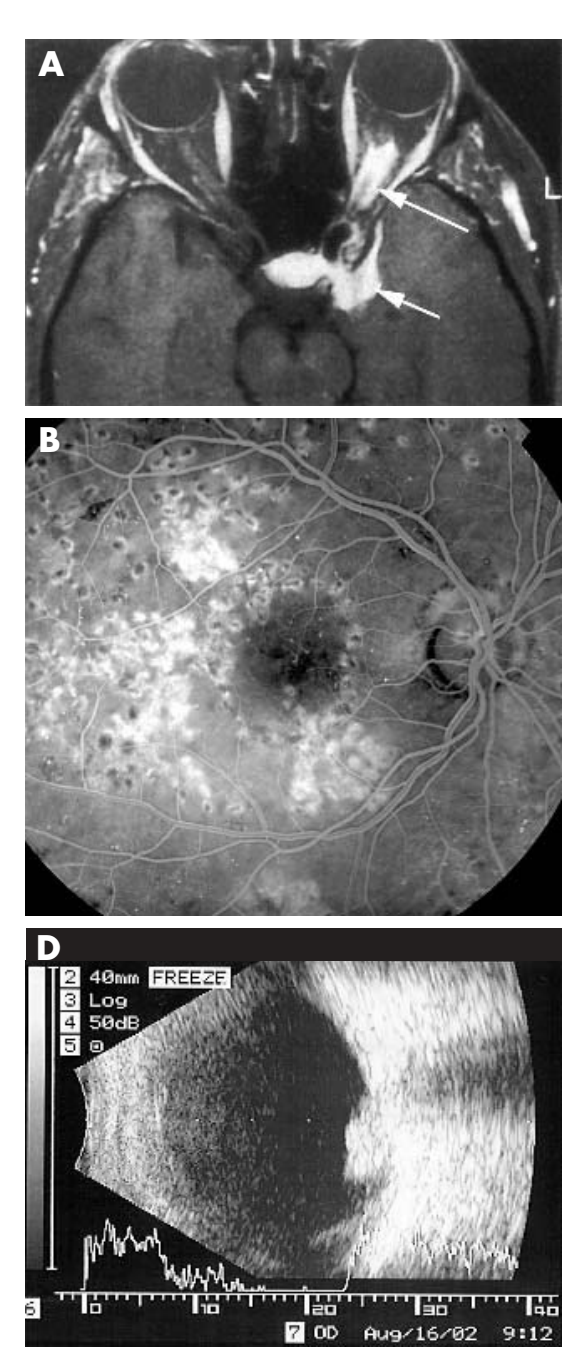

Incidentally, straightforward colour photographs of the optic fundi can be very helpful in certain circumstances. For example, a patient with anomalous discs can be given photographs of their own fundi to show medical practitioners in the future and so potentially prevent unnecessary
Figure 8 Neuro-ophthalmological investigations. (A) Magnetic resonance imaging scan of optic nerve sheath meningioma (arrowed) extending posteriorly through the optic canal to the chiasm. (B) Fluorescein angiogram of diabetic patient showing diabetic retinopathy and laser scars. (C) Normal electroretinogram showing potential detected at the cornea in response to flash of light (courtesy of Dr Graham Holder, Moorfields Eye Hospital).

(D) Orbital ultrasound showing retinal haemangioma in posterior pole of globe.

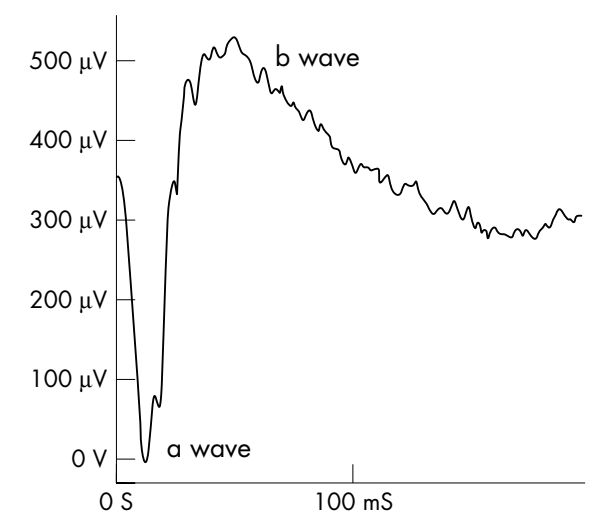


(re)investigation. Similarly, photographic documentation of papilloedema, for example, will facilitate a better assessment of whether a patient is responding to treatment or not.

There are many different variations on the theme of electroretinography which unfortunately cannot be dealt with here. They all rely on the fact that an electrical current is normally detectable from the retina in response to a flash of light. Alterations in this response are seen in a variety of conditions, but the technique is particularly useful in the detection of disturbance of retinal photoreceptor malfunction such as rod or cone dystrophies.

Ocular ultrasound has a relatively limited place in neuroophthalmological practice. It is usually used in the diagnosis and management of conditions such as ocular tumours, retinal detachment, and vitreous haemorrhage. However, it can be used to look at papilloedema, and can be helpful in distinguishing buried optic nerve head drusen from true papilloedema.

Techniques of eye movement recording are discussed in the accompanying article by Danchaivijitr and Kennard. ${ }^{4}$

\section{SUMMARY}

This has been a relatively superficial overview of the various aspects of neuro-ophthalmological examination and investigation. More information is available in the accompanying articles, ${ }^{49}$ but the interested reader is referred to chapters in larger neuro-ophthalmological textbooks. ${ }^{3}{ }^{10}$

\section{...............}

\section{Authors' affiliations}

C J Lueck, The Canberra Hospital and the Australian National University, Canberra, Australia

D F Gilmour, G G Mcllwaine, Princess Alexandra Eye Pavilion and the University of Edinburgh, Edinburgh, UK

\section{REFERENCES}

1 Taylor D, ed. Paediatric ophthalmology, $2^{\text {nd }}$ ed. Oxford: Blackwell Science, 1997.

2 Pandit RJ, Gales K, Griffiths PG. Effectiveness of testing visual fields by confrontation. Lancet $2001 ; 358: 1339-40$.

3 Johnson CA, Keltner JL. Principles and techniques of the examination of the visual sensory system. In: Miller NR, Newman NJ, eds. Walsh \& Hoyt's clinical neuro-ophthalmology, 5th ed. Baltimore: Williams \& Wilkins, 1998.

4 Danchaiviiitr C, Kennard C. Diplopia and eye movement disorders. J Neurol Neurosurg Psychiatry 2004;75(suppl IV):iv24-31.

5 Glaser JS. Neuro-ophthalmology, 2nd ed. Philadelphia: JB Lippincott Co, 1990.

6 Miller NR, Keane JR. Neuro-ophthalmologic manifestations of nonorganic disease. In: Miller NR, Newman NJ, eds. Walsh \& Hoyt's clinical neuroophthalmology, 5th ed. Baltimore: Williams \& Wilkins, 1998.

7 Moseley IF. The plain radiograph in ophthalmology: a wasteful and potentially dangerous anachronism. JR Soc Med 1991;84:76-80.

8 Corbett JJ, Mehta MP. Cerebrospinal fluid pressure in obese subjects and patients with pseudotumor cerebri. Neurology 1983;33:1386-8.

9 Madill SA, Riordan-Eva P. Disorders of the anterior visual pathways. J Neurol Neurosurg Psychiatry 2004;75(suppl IV):iv12-19.

10 Burde RM, Savino PJ, Trobe JD. Clinical decisions in neuro-ophthalmology, 3rd ed. St Louis: Mosby Yearbook, 2002.

11 Miller NR, Newman NJ, eds. Walsh \& Hoyt's clinical neuro-ophthalmology, 5th ed. Baltimore: Williams \& Wilkins, 1998.

- The "neuro-ophthalmological bible". Extensive, comprehensive and well written, this five volume work should be available to all neurologists (both in training and in practice) as a reference work.

12 Burde RM, Savino PJ, Trobe JD. Clinical decisions in neuro-ophthalmology, 3rd ed. St Louis: Mosby Yearbook, 2002.

- An extremely well written and authoritative book dealing with patient investigation and treatment. Structured around presenting symptoms, the text provides a comprehensive guide to patient management. Again, every library should have one.

13 Lee AG, Brazis PW. Clinical pathways in neuro-ophthalmology. New York: Thieme, 1998.

- A good, all round reference text.

14 Jacobsen D, Digre K. http://medlib.med.utah.edu/neuroophth/.

- Library of neuro-ophthalmic video clips prepared by the late Professor Daniel Jacobsen and Professor Kathleen Digre, University of Utah.

15 Hoyt W. http://medstat.med.utah.edu/neuroophth/Hoyt/.

- Professor Bill Hoyt's (as in Walsh and Hoyt, above) collection of slides-website in process of development.

16 North American Neuro-Ophthalmology Society. http:// medlib.med.utah.edu/NOVEL/NOVEL_Updates/.

- NANOS web based education library-currently under development. 